\title{
Young Patients and Robotic Radical Prostatectomy: The Only Way is Up
}

\author{
*Sanchia Goonewardene \\ The Royal Free and UCL, UK \\ Submission: May 26, 2016; Published: October 19, 2016 \\ "Corresponding author: Sanchia Goonewardene, The Royal Free and UCL, NW3 2NG London, UK
}

\begin{abstract}
Prostate cancer within a younger cohort of men continues to be an increasing problem. With a younger population, greater expectations are present, from erectile function, to continence to oncological outcomes. Significant prostate cancer is increasingly diagnosed in younger men, less than 55 years [1]. In a non-screened population young patients are choosing radical surgery for intermediate and high risk disease [1]. This patient group have high expectations with regards to oncological and functional outcomes. The next question arises, what can we do to improve outcomes?
\end{abstract}

Keywords: Robotic radical prostatectomy, outcomes

Abbreviations: ERSPC: European Randomized Study of Screening for Prostate Cancer; AUA: American Urological Association

\section{Mini Review}

Early-onset prostate cancer ( $<55$ years), differs from prostate cancer diagnosed at an older age in several ways [2]. Firstly, among men with high-grade and advanced-stage prostate cancer, those diagnosed at a young age have a higher cause-specific mortality than men diagnosed at an older age [2]. This highlights biological differences between early and lateonset disease [2]. Early-onset prostate cancer also has a strong genetic component. This indicates that young men with prostate cancer could benefit from evaluation of genetic risk [2].When the clinico-pathologic features of men 55 years old or less were examined, it was determined this cohort are more likely to have favourable pathologic characteristics [3]. Yet, screening is not standard.

The results of the European Randomized Study of Screening for Prostate Cancer (ERSPC) trial showed a statistically significant decrease (29\%) in prostate cancer for screened patients, and $23 \%$ negative impact on the life-years gained because of quality of life [4]. This was examined further in young patients [4]. As a result Prostate cancer screening can be costeffective when it is limited to two or three screens between ages 55 to 59 years [4]. American Urological Association (AUA) now no longer recommends testing in men $<55$ years of age without significant risk factors [5]. The argument for this is that harms of PSA-testing including over diagnosis and overtreatment outweigh the benefits [5]. Further supporting this is the fact that aggressive prostate cancer with prostate cancer mortality occurs frequently in this cohort. Failure to screen these men may lead to delay in diagnosis and loss of opportunity for curative treatment.

When examined further, there was no significant difference between the rates of insignificant and high-risk prostate cancer between men $<55$ years and $>55$ years, in either the prostate biopsies or prostatectomy specimens [5].In contrast to this, as part of another cohort, the majority of men $<55$ years of age had Gleason 8-10 prostate cancer on biopsy with a PSA $>4.0$ [6]. As these men are at the highest risk for PCSM, failure to screen younger men may result in a missed opportunity for treatment with curative intent [6]. Additionally, this study demonstrated younger men treated with radiotherapy had worse prostate cancer mortality compared to those treated with radical prostatectomy [6]. Although younger patients may have low-risk disease, the extended life expectancy of these patients exposes them to long-term effects of treatment-related morbidities. Due to their life expectancy, the long-term risk of disease progression leading to death from prostate cancer is increased [2].

There are different methods of treating these patients with radical therapy. Low dose brachytherapy, is one way of treating this cohort. This was reviewed in men aged less than 55 years 


\section{Cancer Therapy \& Oncology International Journal}

with localised prostate cancer [7]. Effective tumour control, with minimal toxicity was demonstrated [7]. The 80 month PSA free survival was $98 \%$ [7]. The median 5-year IPSS and IPSS QoL were 7.5 and 1 respectively [7]. The median IIEF at 5 years was 19 [7]. No secondary malignancies have been reported [7].

When outcomes have been reviewed, results have been encouraging [1]. Despite significant disease in these patients, excellent oncological and functional outcomes are achieved for younger patients [1]. Both erectile function and continence outcomes are acceptable, highlighting the suitability of robotic radical prostatectomy in the management of younger men with prostate cancer [1].

The three long-term goals of radical prostatectomy are cancer control, recovery of urinary continence and sexual function. RARP offers excellent short-term trifecta outcomes when performed by an experienced surgeon [8]. Younger patients demonstrated a shorter time to achieving the trifecta and higher overall trifecta rates when compared to older patients at 6 weeks, 3 months and 6 months after RARP $[9,10]$. However, younger men were more likely to undergo prostatectomy, have lower grade cancer, and equivalent cancer-specific survival at 10 years compared with older men [10]. However, if high risk disease, younger patients had a worse prognosis [10].

\section{Conclusion}

In conclusion, both LDR brachytherapy and robotic radical prostatectomy provide improved outcomes for younger patients diagnosed with prostate cancer.

\section{References}

1. Goonewardene S, Douek N, Brown C, Cahill D, Dasgupta P, et al. (2015) Robotic radical prostatectomy in younger men: Successful outcomes from 236 cases. BJU Int 115: 90.
2. Salinas CA, Tsodikov A, Ishak-Howard M, Cooney KA (2014) Prostate cancer in young men: An important clinical entity. Nat Rev Urol 11(6): 317-323.

3. Kalaw EM, Jara-Lazaro AR, Thike AA, Cheok PY, Geronimo C, et al. (2010) Clinicopathologic features of prostate carcinoma in young men. Proceedings of Singapore Healthcare 19: S172.

4. Heijnsdijk EAM, De Carvalho TMD, Auvinen A, Zappa M, Nelen V, et al. (2015) Cost-effectiveness of prostate cancer screening: A simulation study based on ERSPC data. J Natl Cancer Inst 107(1): dju366.

5. Dantanarayana N, Hossack T, Cozzi P, Brooks A, Lau H, et al. (2015) Men under the age of 55 years have similar prostate cancer pathology as older men: Implications for the AUA screening policy. Journal of Urology 15: 124.

6. Winters B, Holt S, Lin D, Porter M, Cheng H, et al. (2015) Treatment and survival in men under age 55 years diagnosed with gleason 8-10 prostate cancer: Implications of PSA screening. Journal of Urology 193(4): e957.

7. Laing R, Chadwick E, Javed S, Langley SEM (2012) Low dose rate brachytherapy is an excellent treatment option for young men with localised prostate cancer. Radiotherapy and Oncology 103: S93.

8. Samavedi S, Pigilam S, Abdul-Muhsin H, Palmer K, Ebra G, et al. (2014) Continence outcomes robotic assisted radical prostatectomy in suboptimal patients. Journal of Endourology 13(1): e532.

9. Coelho RF, Sivaraman A, Orvieto MA, Chauhan S, Ardila B, et al. (2010) Continence, potency and oncologic outcomes after robotic-assisted radical prostatectomy: The search for the trifecta - Early results. European Urology Supplements 3: 501.

10. Lin DW, Porter M, Montgomery B (2009) Treatment and survival outcomes in young men diagnosed with prostate cancer: A populationbased cohort study. Cancer 115(13): 2863-2871.

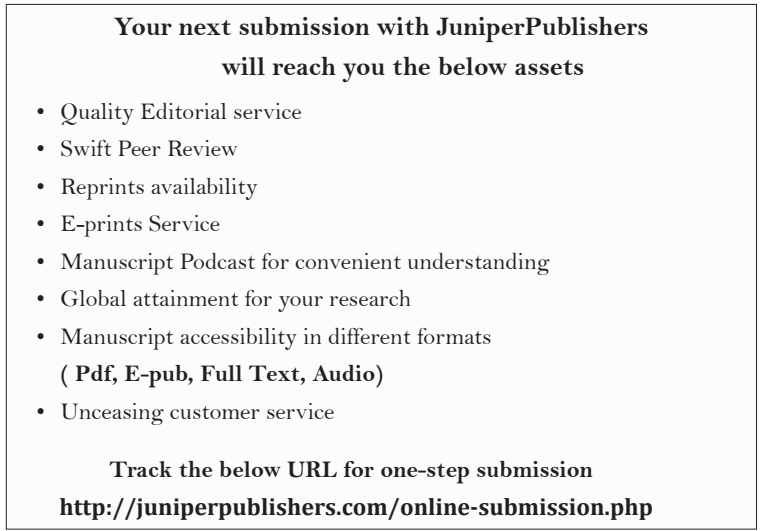

